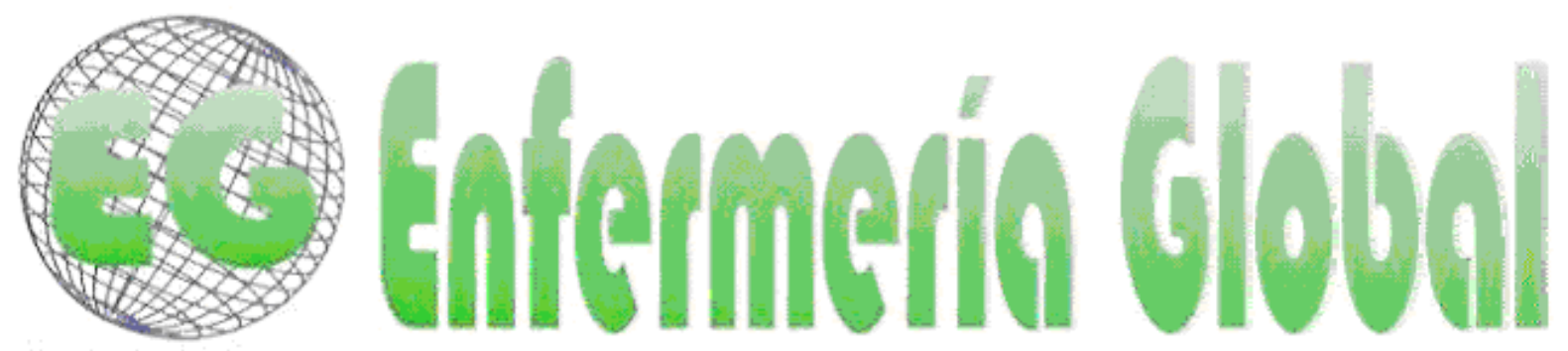

\title{
Valoración de la adherencia en el seguimiento del paciente diabético y uso de lenguaje enfermero
}

Valuation of the adhesion in the follow-up of the diabetic patients and the use of the nurse language

\section{*Del Castillo Arévalo, Fernanda *Salido González, Margarita *Losada García, Adamina *García García, Mercedes *Fernández Prieto, Teresa *Blanco Gutiérrez, Mª Luz}

*Enfermeras de Atención Primaria del Centro de Salud de Contrueces, perteneciente al Área Sanitaria V de Asturias, SESPA. E-mail: fcastia@gmail.com

Palabras clave: Adherencia farmacológica; taxonomías enfermeras; diabetes; atención primaria.

Keywords: Patient adherence; taxonomies nurses; diabetes; primary care

\section{RESUMEN}

Mejorar la baja adherencia terapéutica de los pacientes crónicos, aumentar la seguridad del paciente y la efectividad del sistema sanitario; las enfermeras tienen en ello una función importante y disponen de un lenguaje propio para denominar esta situación.

Objetivos: Conocer el número de pacientes diabéticos que durante el año 2011, acudieron a la consulta de su enfermera; si éstas les valoraron la adherencia farmacológica y dieron consejo sobre medicación, alimentación y ejercicio físico. Saber si se expresa mediante lenguaje enfermero.

Método: Estudio descriptivo transversal. Población a estudio: diabéticos que acuden a consulta de enfermería durante el año 2011. Datos extraídos de OMI-AP y revisión de historias clínicas. Variables expresadas como frecuencias absolutas y porcentajes.

Resultados: Pacientes diabéticos 719, acudieron a consulta de enfermería 465 (64,6\%). Tienen recogido consejo sobre alimentación $369(80,7 \%)$ y sobre ejercicio $307(67 \%)$. Tienen tratamiento farmacológico $390(85,3 \%)$, recogida la adherencia farmacológica $336(72,2 \%)$, consejo "uso adecuado de medicación" 209 (45\%). La NIC 5616 "Enseñanza medicamentos prescritos", figura en 27 pacientes y 16 tienen planes de cuidados en ese periodo.

Conclusiones: La valoración de la adherencia farmacológica es una actividad integrada por las enfermeras en el seguimiento de los pacientes diabéticos. El consejo sobre alimentación se realiza de forma habitual. Se debe incrementar el consejo sobre ejercicio físico y uso adecuado de medicamentos. La utilización de lenguaje enfermero no está incorporada en nuestra práctica enfermera. Lo facilitaría mejoras en la herramienta informática y que formara parte de los objetivos de las gerencias. 


\section{ABSTRACT}

Improving the low treatment adherence in chronic patients increases patient 'security and effectiveness of the health system. Nurses play an important role in this objective and they have their own language to describe this situation.

Goals: To know the number of diabetic patients that came to the nurse consultancy throughout the year 2011; whether nurses valued their pharmacologic adherence and if they gave advise about medication, nutrition and physical exercise. Another goal was to know if all this information was expressed using nurse language.

Methods: It is a cross-sectional descriptive study. Population of study: Diabetic patients that came to the nurse consultancy throughout the year 2011. Data were acquired from OMI-AP and review of medical records. Variables were expressed as absolute frequencies and percentages.

Results: From a total of 719 diabetic patients, $465(64,6 \%)$ attended the nurse consultancy. 369 (80, $7 \%)$ received advice about nutrition and 307 (67\%) about physical exercise. 390 (85, 3\%) were under pharmacological treatment, in $336(72,2 \%)$ pharmacologic al adherence was registered, the advice: "adequate use of medication" was present in 209 (45\%). NIC 5616 "Teaching on prescribed drugs" appeared in 27 patients and 16 had care plans in that period.

Conclusions: Evaluation of pharmacologic adherence is an activity included in the follow up made by nurses in diabetic patients. Advice about nutrition is usually given. Advice about physical exercise and adequate use of medication must be reinforced. The use of nurse language is not incorporated in our nurse practicing. This would be facilitated by improving informatics tools and incorporating this item in management strategy.

\section{INTRODUCCIÓN}

La Organización Mundial de la Salud ha definido de forma general la adherencia, como el grado en que el comportamiento de una persona - tomar su medicación, seguir un régimen alimentario, realizar cambios del modo de vida- se corresponde con las recomendaciones de un prestador de asistencia sanitaria.

La falta de adherencia al tratamiento o incumplimiento terapéutico es un problema relevante, con mayor importancia en el tratamiento de las enfermedades crónicas. La OMS estima que el cumplimiento farmacológico en estas patologías es de un $50 \%$ en los países occidentales. ${ }^{1}$. Otros estudios ${ }^{2,3}$ han demostrado que en los países desarrollados el cumplimiento terapéutico en pacientes crónicos se sitúa entre el 50 y el $75 \%$.

En el citado informe de la OMS, se ponen de relieve algunos aspectos importantes como que: "Aumentar la efectividad de las intervenciones sobre cumplimiento terapéutico puede tener una repercusión mucho más grande sobre la salud de la población, que cualquier mejora de los tratamientos médicos específicos", que "mejorar la adherencia terapéutica mejora la seguridad del paciente y la efectividad del sistema sanitario" y que el cumplimiento terapéutico requiere un enfoque multidisciplinario, en el que los profesionales de la enfermería pueden desempeñar una función importante en las intervenciones orientadas a los pacientes al educarlos y facilitar la adherencia al tratamiento ${ }^{1}$.

Entre las diferentes enfermedades crónicas, tiene especial relevancia la diabetes por causar alta morbilidad y mortalidad. Su prevalencia en nuestro país según datos del Ministerio de Sanidad es del 6,6\% en la población entre 30 y 65 años y del $16,7 \%$ en los mayores de 65 años $^{4}$, aunque se cree que estos datos han sido superados 
llegando a estimarse una prevalencia en edad adulta entre el $10-15 \%{ }^{5}$. Estos pacientes ocupan un gran número de las visitas a médicos y enfermeras de familia, debiendo establecerse una buena coordinación entre ambos para un adecuado seguimiento ${ }^{6,7}$. El manejo de la adherencia, su valoración, prevención y abordaje ante el incumplimiento, es una responsabilidad multiprofesional, y debería estar presente en todas las consultas de seguimiento de los pacientes con patologías crónicas.

Las enfermeras disponen de lenguajes estandarizados; la utilización de las taxonomías NANDA-NIC-NOC contribuye a normalizar y unificar el lenguaje enfermero. Para describir las conductas del paciente relativas al cumplimiento de las indicaciones terapéuticas tenemos los diagnósticos enfermeros "Gestión Ineficaz de la propia salud"8, que se define como "patrón de regulación e integración en la vida diaria de un programa de tratamiento de la enfermedad y de sus secuelas que resulta insatisfactorio para alcanzar objetivos específicos de salud" e "Incumplimiento", definido como "Conducta de una persona o un cuidador que no coincide con el plan terapéutico o de promoción de la salud acordado entre la persona (o la familia o la comunidad) y un profesional de la salud. Ante un plan terapéutico o de promoción de la salud acordado, la conducta de la persona o del cuidador lo incumple total o parcialmente y puede conducir a resultados clínicos parcial o totalmente ineficaces"; estos diagnósticos aparecen en algunos artículos entre los diagnósticos enfermeros más utilizados ${ }^{9,10}$ o priorizados por importancia percibida ${ }^{11}$ en Atención Primaria (AP).

Una de las intervenciones NIC recomendadas para estos diagnósticos es La NIC $5616^{12}$ "Enseñanza medicamentos prescritos", cuya definición es: "Preparación de un paciente para que tome de forma segura los medicamentos prescritos, y observar sus efectos". Las actividades que se incluyen en esta intervención son realizadas cotidianamente por las enfermeras, por ello debiera usarse de forma habitual en las consultas de AP; su uso está recomendado en diversas guías de atención a personas con diabetes ${ }^{10,13,14}$

En este trabajo nos planteamos conocer cuál es el grado de cumplimiento de las enfermeras de nuestro Centro de Salud ante las recomendaciones de valoración de la adherencia y del uso de los lenguajes enfermeros en el seguimiento de las personas con diabetes.

\section{OBJETIVOS}

El objetivo de este estudio es conocer el número de pacientes diabéticos que acudieron a la consulta de su enfermera de familia durante el año 2011, y si éstas les valoraron la adherencia farmacológica y dieron consejo sobre uso adecuado de la medicación. Conocer si les dieron consejo sobre alimentación adecuada y ejercicio físico. Saber si se expresan mediante lenguaje enfermero.

\section{MATERIAL Y MÉTODO}

Diseño: Estudio descriptivo transversal.

Población a estudio: pacientes con diabetes tipo II pertenecientes a cinco cupos del Centro de Salud de Contrueces, Gijón, que acudieron a la consulta de su enfermera durante el año 2011.

Criterios de exclusión: pacientes que fueron éxitus durante ese año. 
Los datos son extraídos a través de OMI-AP por el Área de Sistemas del Servicio Regional de Salud; posteriormente se realiza una revisión de las historias clínicas de los pacientes incluidos en nuestra revisión, valorando la existencia de registro de los ítems considerados como las variables a estudio. Dichas variables son: Adherencia farmacológica. Consejo: Uso adecuado de medicación. Consejo sobre alimentación. Consejo sobre ejercicio.

La valoración del uso de lenguaje enfermero se centra en la existencia de Planes de Cuidados abiertos en estos pacientes durante el periodo de estudio y del registro de la NIC: Enseñanza medicamentos prescritos.

Se presentan las variables como frecuencias absolutas y porcentajes.

\section{RESULTADOS}

El número de pacientes con diagnóstico de diabetes de los cupos estudiados es de 719, de ellos han acudido durante el año 2011 a la consulta de enfermería 465 personas, lo que supone el $64 \%$.

Se excluyen de la revisión 8 por ser éxitus.

Tienen tratamiento farmacológico 390 (85,3\%).

De los 465 pacientes que acudieron a la consulta de enfermería se valoró la adherencia a 336 , lo que supone el $72,2 \%$.

Han recibido consejo sobre uso adecuado de medicación 209 (45\%).

De la población total analizada de 457 pacientes, tienen recogido que se les ha dado consejo sobre alimentación a $369(80,7 \%)$ y consejo sobre la realización de ejercicio a 307 (67\%).

Tienen planes de cuidados abiertos en el periodo de estudio 16 personas y en 27 figura la NIC 5616 Enseñanza medicamentos prescritos

\section{DISCUSIÓN}

El primer aspecto a destacar es que solo el $64 \%$ de los pacientes diabéticos han acudido a la consulta de su enfermera. El seguimiento de los pacientes con diabetes debe ser coordinado entre médico y enfermera ${ }^{6,7}$. Existen evidencias en la literatura de que la implicación de las enfermeras mejora la calidad de los cuidados a los pacientes diabéticos ${ }^{8,9,15}$. Se destaca que la enfermera debe ostentar un papel fundamental en la atención de las personas con diabetes, correspondiéndole las actividades relacionadas con la educación, el control y el seguimiento del paciente, siendo la evaluación del cumplimiento uno de los contenidos de la consulta de enfermería $6,7,16,17,18$

En nuestro estudio no valoramos cómo es el cumplimiento de los pacientes al tratamiento farmacológico ni a las medidas terapéuticas de dieta y ejercicio, sino si las 
enfermeras lo evalúan y aconsejan sobre ello, por tanto, no podemos ofrecer resultados del cumplimiento de los pacientes.

El grado de cumplimentación por las enfermeras de las variables a estudio, en general, nos parece ajustado aunque deficitario para el consejo sobre uso adecuado de medicamentos.

El que un $72,2 \%$ de los pacientes tengan valorada la adherencia en la consulta de la enfermera, nos parece un dato relevante, aunque mejorable, dado que uno de los contenidos de la visita de enfermería debe ser la evaluación del cumplimiento ${ }^{6,7,17}$; se cree que la consulta de enfermería constituye un entorno ideal para el abordaje de la adherencia dado las competencias de estos profesionales en la educación sanitaria y se piensa que las enfermeras tendrían facilidad para la obtención de información sobre miedos y reticencias de cada paciente ${ }^{19}$.

En cambio, existen discrepancias en la importancia que las propias enfermeras otorgan a la adherencia farmacológica ${ }^{20}$, por lo general prestan atención al cumplimiento farmacológico, pero algunas manifiestan que la revisión de los medicamentos que tiene prescritos el paciente y el seguimiento de cómo los toma, es una responsabilidad médica y que las enfermeras deben centrarse en el seguimiento de las indicaciones terapéuticas basadas en medidas higiénico-dietéticas, como el seguimiento de una alimentación adecuada y la práctica de ejercicio de forma regular. Estas opiniones parecen corresponderse con el que en nuestro trabajo se observe un mayor porcentaje del consejo sobre alimentación y ejercicio que sobre el uso adecuado de fármacos.

Una debilidad de nuestro estudio es que no se conoce el método utilizado por las enfermeras para evaluar la adherencia; en el periodo a estudio solo se registraba mediante un check list, en el que se marcaba SI- No, a si el paciente es adherente. Una de las quejas que exponen las enfermeras de nuestra Área Sanitaria a la hora de valorar la adherencia es la ausencia de un protocolo específico que facilite efectuar esta actividad, refieren formular preguntas directas, y de fácil respuesta, del tipo: "¿se le olvida a Ud. tomar alguna vez las pastillas?" Otros métodos utilizados son el juicio clínico y la falta de control del proceso ${ }^{20}$.

En diversos artículos se hace referencia, aunque sin cuantificar, a la escasa valoración que realizan los profesionales sanitarios del cumplimento de los pacientes de las recomendaciones recibidas y refieren que cuando lo hacen suele ser de forma general, sin utilizar la mejor metodología para ello ${ }^{21,22,23}$

Otro dato a considerar es que el consejo sobre "uso adecuado de medicación" consta que se ha impartido al $45 \%$ de los diabéticos. Es necesario un buen conocimiento por parte de las enfermeras de los fármacos más comúnmente utilizados en estos pacientes, dosis, horarios, efectos secundarios, qué hacer si éstos se presentan, etc, para poder informarles adecuadamente, así mismo, es preciso que se incorpore como una práctica ineludible de las consultas de seguimiento, independientemente de que se considere que el paciente es buen cumplidor. Si en el conocimiento de fármacos las enfermeras demandan más formación ${ }^{20}$, también es necesaria la formación en la evaluación y mejora de la adherencia ${ }^{24}$ 
En cuanto a los consejos sobre alimentación y ejercicio: Hemos visto que de los 457 pacientes, tienen recogido que se les ha dado consejo sobre alimentación a 369 $(80,7 \%)$ y consejo sobre la realización de ejercicio a 307 (67\%).

La realización del consejo sobre alimentación parece estar muy interiorizada por las enfermeras dado el alto porcentaje de pacientes que lo han recibido.

Los objetivos del tratamiento dietético en la diabetes son lograr un peso adecuado, el control glucémico y el control de factores de riesgo cardiovascular. Existe evidencia científica con un grado A de Recomendación para: Programas estructurados con asesoramiento dietético, reducción de la ingesta de grasa ( $<30 \%$ de energía diaria), contenidos de hidratos de carbono entre $55 \%-60 \%$ de la energía diaria y consumo de fibra de 20-30 g. En pacientes con un IMC $\geq 25 \mathrm{~kg} / \mathrm{m} 2$ la dieta debe ser hipocalórica ${ }^{6,17}$. No tenemos constancia del tipo de recomendación efectuada en nuestro Centro de Salud; aunque las enfermeras han realizado diversos cursos de formación en diabetes con un amplio número de horas dedicado a la alimentación, no existe un protocolo o consenso en el centro de cómo debe ser el asesoramiento nutricional a impartir.

Observamos que el ejercicio se aconseja en menor medida que las recomendaciones nutricionales

Según la evidencia disponible, se recomienda la realización de ejercicio físico regular y continuado, de intensidad aeróbica 0 anaeróbica, o preferiblemente una combinación de ambas. La frecuencia recomendada es de tres sesiones semanales en días alternos, progresivas en duración e intensidad, y preferiblemente supervisadas. Grado de Recomendación $A^{6,17}$

Pensamos que la formación para la recomendación del ejercicio adecuado es más deficitaria y que existe menor conocimiento sobre la intensidad requerida, cómo medirla, tipo de ejercicio apropiado a cada paciente en función de sus características individuales y de la coexistencia de otras patologías. La formación en este campo se ha impulsado en menor medida que otros aspectos del tratamiento de estos pacientes.

La adherencia es una conducta, no un rasgo de la personalidad, así un paciente puede ser adherente por un periodo de tiempo y más tarde dejar de serlo, o adherirse a unas indicaciones y no a otras; de ahí la necesidad de sistematizar la forma de medir y registrar la adherencia y dado que los pacientes suelen cumplir mejor los tratamientos con fármacos que otro tipo de medidas es necesario evaluar la cumplimentación del tratamiento farmacológico y del no farmacológico ${ }^{25}$.

La valoración de las distintas partes del tratamiento debe realizarse de forma individualizada. El estudio $\mathrm{DAWN}^{26}$, refiere que el cumplimiento en pacientes diabéticos tipo II al tratamiento farmacológico es del $78 \%$, a la dieta del $37 \%$, y al ejercicio del $35 \%$, se confirma un mejor cumplimiento del tratamiento farmacológico que de la terapia nutricional y el ejercicio físico.

Se estima que el uso del ordenador en las consultas puede ayudar a investigar el cumplimiento $^{23}$. El que haya un ítem sobre este aspecto en la carpeta de registro del seguimiento de pacientes con procesos crónicos en nuestra Comunidad Autónoma puede haber favorecido un cumplimiento bastante alto por parte de las enfermeras. 
En un estudio en nuestro país sobre los registros de diabetes en la historia informática, se demuestra que la Historia Clínica electrónica mejora los indicadores de calidad de la atención al paciente diabético; encuentran que en general existe un moderado registro del plan terapéutico relacionado con la diabetes $(69,1 \%)$. Diez Comunidades registran todas las variables analizadas (dieta, ejercicio físico, cumplimiento y autoanálisis), Los datos sobre dieta, ejercicio físico y autoanálisis tienen idéntica implantación (70,5\%), siendo menor el registro sobre cumplimiento terapéutico $(64,7 \%)^{27}$. Nuestros datos no difieren demasiado de los encontrados en el estudio citado.

Existen numerosas estrategias para promover la adherencia, aunque no hay evidencias concluyentes, sí parece confirmarse que lo más efectivo es la combinación de varias de ellas y la individualización de cada paciente ${ }^{28,29}$. La información oral y escrita es una de las estrategias recomendadas. Las enfermeras consideran que la falta de información de los pacientes sobre su enfermedad y los fármacos prescritos puede dar lugar a errores en la comprensión de las instrucciones y por tanto a un seguimiento incorrecto de las mismas ${ }^{30}$. En un estudio de EEUU se observó que el 32,5 de los diabéticos no eran adherentes y más del $50 \%$ de los pacientes no contaba con conocimientos suficientes acerca de su situación ${ }^{31}$; las enfermeras para paliar estas carencias refieren procurar proporcionarles información verbal y escrita ${ }^{30}$; estas ideas pueden explicar algunos de los resultados encontrados en cuanto a la emisión de consejo sobre los diversos aspectos.

Los datos más frustrantes son los relativos al uso del lenguaje enfermero, solo el $6,9 \%$ de los pacientes estudiados tenían la etiqueta NIC "Enseñanza medicamentos prescritos" y 16 tenían un plan de cuidados abierto en ese periodo, lo que supone el $4,1 \%$ de la población estudiada. Esto da una clara idea de que el uso de lenguajes enfermeros no está interiorizado en la cotidianeidad de la actuación de las enfermeras de nuestro Centro de Salud.

El concepto diagnóstico "Gestión ineficaz de la propia salud" responde apropiadamente a uno de los principales problemas de las personas con diabetes que es el correcto autocuidado y un buen manejo del tratamiento, incluyendo fármacos y medidas relativas a estilos de vida. Si las enfermeras, como se ha visto, evalúan el cumplimiento de las indicaciones terapéuticas es una inconsistencia que no utilicen su propio lenguaje para expresarse.

En el año 2005, se emitieron en el Centro de Salud donde se realiza el estudio 1680 diagnósticos enfermeros (datos internos de la gerencia del Área Sanitaria), de ellos $276(16,4 \%)$ eran relativos al cumplimiento del tratamiento, estos datos contrastan con los actuales, aunque en el 2005 no estaban circunscritos a los pacientes con diabetes, extrapolándolo nos dan idea de un porcentaje muy superior de diagnósticos emitidos. Pensamos que es debido a que en ese momento se estaba realizando un proceso de implantación de la metodología enfermera y constituía uno de los objetivos de la gerencia. Otro hecho importante es que se comunicaba a las enfermeras los resultados obtenidos; la retroalimentación (feedback) de la información de los indicadores que se evalúan es la base para mejorar la práctica clínica ${ }^{32}$ y reforzar la actividad a implementar.

Una posible justificación puede ser que habitualmente las enfermeras de nuestro centro registran la actividad de estos pacientes en una carpeta diseñada para el 
seguimiento por enfermería de los enfermos crónicos que incluyen los ítems a estudio pero no diagnósticos NANDA ni intervenciones NIC.

Las intervenciones NIC se usan de forma regular para otro tipo de actividades pero no cuando se hace el seguimiento de estos pacientes.

Otra dificultad es que resulta trabajoso el registro de planes de cuidados en nuestro programa informático (OMI-AP), además no se realizan los reajustes necesarios que concuerden con las actualizaciones de la NANDA; dichas actualizaciones tampoco contribuyen a una adherencia de las enfermeras con el lenguaje propio ya que algunos cambios tienen difícil explicación.

Recientemente se han incorporado en nuestra Comunidad Autónoma unos protocolos informáticos para el paciente cardio-metabólico que incluyen planes de cuidados estandarizados, siendo uno de los diagnósticos enfermeros seleccionados el de Gestión ineficaz de la propia salud; esto podría facilitar la emisión de etiquetas diagnósticas en las consultas de las enfermeras, lo que no quiere decir que se interiorice el lenguaje.

\section{CONCLUSIONES}

La valoración de la adherencia farmacológica es una actividad incorporada por las enfermeras de nuestro Centro de Salud en el seguimiento de los pacientes con diabetes aunque habría que mejorar y sistematizar la forma de evaluarla.

El consejo sobre alimentación se realiza de forma habitual en estos pacientes.

Se debe reforzar en las enfermeras la necesidad de dar consejo sobre ejercicio y sobre el uso adecuado de los medicamentos.

No está incorporado el lenguaje enfermero. La utilización de lenguaje enfermero a través de las taxonomías NANDA-NIC-NOC no está incorporada en nuestra práctica enfermera. Creemos que lo facilitaría si formara parte de los objetivos de las gerencias, se evaluara periódicamente y revertiera la información a los profesionales o que éstos tuvieran la capacidad de poder evaluar por sí mismos su trabajo, también que se realizaran mejoras y actualizaciones periódicas en la herramienta informática.

\section{BIBLIOGRAFÍA}

1- Sabate E. Adherencia a los tratamientos a largo plazo. Pruebas para la acción (Documento OMS traducido). Ginebra: Organización Mundial de la Salud 2004. Consulta: 28 de marzo 2012. Disponible en http:// www.paho.org/Spanish/AD/DPC/NC/adherencia-largo-plazo.pdf

2- World Health Organization (WHO). Adherence to Long-term Therapies.2003. Disponible en: http://www.who.int/chp/knowledge/publications/adherence_report/en/

3- DiMatteo MR. Variations in patients adherence to medical recommendations: a quantitative review of 50 years of research. Med Care.2004;42:200-9.

4- Estrategia en diabetes del Sistema Nacional de Salud. Madrid: Ministerio de Sanidad y Consumo. Centro de publicaciones; 2007.

5-Valdés S, Rojo-Martínez G, Soriguer F. Evolución de la prevalencia de la diabetes tipo 2 en población adulta española. Med Clin (Barc). 2007; 129(9): 352-5 
6- Guía de práctica clínica sobre diabetes tipo 2. Guías de práctica clínica en el SNS. Ministerio de Sanidad y Consumo. Vitoria, 2008

7- LA MEJORA ASISTENCIAL DEL DIABÉTICO. La calidad asistencial y los programas de mejora en diabetes. Sociedad Española de Diabetes (SED), 2009 http://www.sediabetes.org/gestor/upload/SED LaMejoraAsistencialDelDiabetico\%282 \%29.pdf

8- NANDA International. Diagnósticos enfermeros: Definiciones y Clasificación, 20092011. Madrid, Elsevier 2010.

9- Maña M, Fernández E, Veledo P, García P, González A, Soto L, Mesas A. Enfermeras centinelas en red. Consulta: 12 Marzo 2009. http://www.enfermeriacomunitaria.org/documentos/bec/bec 27/centinela.pdf

10- Morilla-Herrera J. C., Morales-Asencio J. M., Fernández-Gallego M. C., Berrobianco Cobos E., Delgado Romero A.. Utilidad y validez de un instrumento basado en indicadores de la Nursing Outcomes Classification como ayuda al diagnóstico de pacientes crónicos de Atención Primaria con gestión ineficiente de la salud propia. Anales Sis San Navarra [revista en la Internet]. 2011 Abr [citado 2012 Jun 30] ; 34(1): 51-61. Disponible http://scielo.isciii.es/scielo.php?script=sci_arttext\&pid=S1137-

66272011000100006\&lng=es. http://dx.doi.org/10.4321/S1137-66272011000100006. 11- Brito Brito PR. Diagnósticos enfermeros priorizados en atención primaria. Enfermería Global., Murcia, n. 16, jun. 2009. Consulta: 28 marzo 2012. Disponíble en http://scielo.isciii.es/scielo.php?script=sci_arttext\&pid=S1695-

$61412009000200008 \&$ Ing $=$ pt\&nrm=iso

12- Bulechek G, Butcher $\mathrm{H}$, McCloskey J. Clasificación de Intervenciones de Enfermería (NIC). Elsevier, 5a ed. Barcelona, 2009.

13- Guía de Atención Enfermera a Personas con Diabetes. Servicio Andaluz de Salud Dirección General de Asistencia Sanitaria. Dirección Regional de Desarrollo e Innovación en Cuidados. 2a Edición revisada, 2006. ISBN: 84-689-9734-X.

14- Anexo al Plan Integral de Diabetes Mellitus de Castilla-La Mancha. 2007-2010. Dirección General de Ordenación y Evaluación. Consejería de salud y bienestar social. Junta de comunidades de Castilla-La Mancha.

15- Martínez Pastor A, Leal Hernández M, Martínez Navarro A, Navarro Oliver A, Lifante Pedrola Z, Gómez Ruiz M. Efectos de un programa de revisión en el control de los diabéticos tipo 2 seguidos en atención primaria. Programa Diabetes First. Endocrinol Nutr. 2010;57(1):16-21

16- Guía de diabetes para Atención Primaria. Samfyc, 2010. Consultado marzo 2012. http://www.grupodiabetessamfyc.cica.es/index.php/guia-clinica.html

17- Práctica clínica en la DM2. Análisis crítico de las evidencias por la redGDPS. 2011 redGDPS y Elsevier. Consultado marzo 2012. http://www.redgdps.org/gestor/upload/file/guias/guia gedaps practica-cinica-2010.pdf

18- Atención de enfermería al paciente diabético. Consejo General de Enfermería. Noviembre 2010.

19- Adherencia al tratamiento farmacológico en patologías crónicas. Infac. Vol.19 • Nº 1, 2011. Consultado marzo 2012. http://www.osanet.euskadi.net/cevime/es

20- Rodríguez García, MJ; del Castillo Arévalo, F. Enfermeras de Atención Primaria opinan, analizan y proponen mejoras en el manejo de la adherencia farmacológica. Enfermería Global. 25, enero 2012, ISSN 1695-614. Consultado marzo 2012. http://scielo.isciii.es/scielo.php?pid=S1695-61412012000100012\&script=sci_arttext 21- Márquez Contreras, E. Evaluación del incumplimiento en la práctica clínica. Hipertensión. 2008;25:205-13. 
22- Artigas García R, Badiola Villa $\mathrm{H}$, Segrelles Lloret M Estudio del incumplimiento terapéutico en la hipertensión arterial. SEMG, 2010 en http://www.medicinageneral.org/revista_126/pdf/113-117.pdf

23- ¿Nos puede ayudar el ordenador como método diagnóstico del incumplimiento farmacológico, en las consultas médicas y de enfermería? Grupo de Cumplimiento de la Asociación de la Sociedad Española de Hipertensión y Liga Española para la Lucha Contra la Hipertensión Arterial. Cumplimiento terapéutico en la HTA. Editorial. Actualizaciones. Vol. 2. Núm. 3. Septiembre 2005.

24- Fernández, L; Guerrero, L; Segura, J; Gorostidi, M. Papel del personal de enfermería en el control de la hipertensión arterial y en la investigación cardiovascular. Hipertens riesgo vasc. 2010;27(Supl 1):41-52

25- Jansà $M$, Vidal $M$. Métodos directos e indirectos para medir el cumplimiento no farmacológico http://sediabetes.org/gestor/upload/revistaAvances/25-1.pdf\#page=55 Importancia del cumplimiento terapéutico en la diabetes mellitus. Av. Diabetol. 2009; 25: 55-61

26- (Peyrot M, Rubin RR, Lauritzen T, Snoek FJ, Matthewa DR, Skovlund SE. Psychological problems and barriers to improved diabetes management. Results of the Cross National Diabetes, Attitudes Whishes and Needs Whishes and Needs (DAWN) Study. Diabet Med. 2005;22:1379-85)

27- Navarro-Pérez J, et al. La historia clínica electrónica y los registros sobre diabetes en España. Av Diabetol. 2011. doi:10.1016/j.avdiab.2011.09.001 http://www.elsevier.es/sites/default/files/elsevier/eop/S1134-3230\%2811\%2900002-

$\underline{0 . p d f}$

28- Haynes RB, Ackloo E, Sahota N, McDonald HP, Yao X. Intervenciones para mejorar la adherencia a la medicación (Revisión Cochrane traducida). En: La Biblioteca Cochrane Plus, número 3, 2008. (Traducida de The Cochrane Library, Issue. Chichester, UK: John Wiley \& Sons, Ltd.).

29- Gago López M. Evidencias en cumplimiento terapéutico. Enfermería basada en la evidencia. Revista electrónica de Portales Médicos. Publicado: 16/08/2010 http://www.portalesmedicos.com/publicaciones/articles/2377/1/Evidencias-encumplimiento-terapeutico.-Enfermeria-basada-en-la-evidencia. Consultado 2 de julio 2012.

30- Rodríguez-García MJ, Castillo-Arévalo F. Enfermeras y adherencia farmacológica: discurso y conducta. Index Enferm [revista en la Internet]. 2011 Sep [citado 2012 Jun 30] ; 20(3): 160-164. Disponible en: http://dx.doi.org/10.4321/S113212962011000200005 .

31- Bailey CJ, Kodack M. Patient Adherence to Medication Requirements for Therapy of Type 2 Diabetes. International Journal of Clinical Practice 65(3):314-322, Mar 2011 32- Jamtvedt G, Young JM, Kristoffersen DT, O'Brien MA, Oxman AD. Efectos de la auditoría y la retroalimentación (feedback) en la práctica profesional y las medidas de resultado de atención sanitaria. La Biblioteca Cochrane Plus.2011 Número 1 ISSN $1745-9990$ 